\title{
Design of a Novel CRLH-TL Structure
}

\author{
Siwen Sun • Shanjia Xu
}

Received: 19 November 2006 / Accepted: 3 August 2010 /

Published online: 17 August 2010

(C) The Author(s) 2010. This article is published with open access at Springerlink.com

\begin{abstract}
Two new types of interdigital capacitors (IDC) are proposed to be used in a novel Composite Right/Left-Handed Transmission Line (CRLH-TL) structure. The LC parameters of the traditional and novel IDC are respectively extracted for comparisons with Ansoft HFSS9 full-wave simulation. In contrast with traditional structure, such two new IDC enhance the capacitance values dramatically by about $38.7 \%$ and $100 \%$, respectively, compared with the traditional structure within the same size of etched area. Also dispersion relationships are calculated by both simulation and Bloch theorem. A one dimension (1-D) CRLH-TL structure consisting of such new IDC and meandering grounded inductors is designed, modeled and simulated. The presented structure is able to operate within a much lower and wider Left-handed (LH) frequency band than traditional ones.
\end{abstract}

Keywords CRLH-TL structure $\cdot$ LH operating frequency $\cdot$ Interdigital capacitors

\section{Introduction}

Left-handed materials (LHM), also named negative index materials (NIM) for their negative refraction index resulting from simultaneously negative real parts of electric permittivity and magnetic permeability, were firstly expected and theoretically investigated by Russian Physicist Veselago in late 1960 [1]. Three decades later, Smith et al. fabricated the first LHM using a periodic array of split-ring resonators (SRRs) combined with an array of straight conducting wires[2], on the basis of Pendry's theoretical work in [3, 4]. Since then, LHM has highly attracted both scientific and engineering communities for its extraordinary characters which are not generally found in nature. In 2003, Parazzoli experimentally verified and simulated negative refraction index of LHM using Snell's Law

Supported by the National Natural Science Foundation of China (No. 60171037) (No. 60371010) and (No. 63531020)

S. Sun $(\bowtie) \cdot$ S. Xu

Department of Electronic Engineering and Information Science,

University of Science \& Technology of China, Hefei, People's Republic of China, 230027

e-mail: seawensun@gmail.com 
[5]. Subsequently, three different groups [6-8] almost at the same time introduced LHM concept into transmission-line (TL) and realized LH-TL by loading a TL with lumped elements or distributed inductors and capacitors. However, because of unavoidable parasitic series inductance and shunt capacitance which will result in a right-handed properties' contribution with the frequency increasing, the LH-TL is actually a CRTH-TL structure [6]. In CRTH-TL, previous stop-band in low frequency region can be switched to pass-band because negative or backward wave propagation appears for anti-parallel phase and group velocities in Left-handed band. In regard of this, it is promising to apply CRLH-TL in microwave and millimeter wave devices for reducing physical length. So far, many novel microwave resonators, antennas, filters, couplers and etc. have been developed with periodical or nonperiodical CRLH-TL structure [9-11]. In this paper, we continue to explore the potential of CRLH-TL and focus on enhancing the left-handed nature and thereby pushing down the LH frequency band while keeping the unit-cell size unchanged.

This paper is organized as follows. In Section 2, two new IDC evolving from traditional structure are presented. Then equivalent circuit model is established and distributed LC parameters are extracted based on the results from full-wave simulation. Related analysis is also discussed in this section. In Section 3, a 1-D CRLH-TL unit structure composed of such new IDC is designed, modeled and simulated. Frequency response and dispersion diagram are given. Finally, the main conclusions of this paper are highlighted in Section 4.

\section{New types of interdigital capacitors}

\subsection{Implementation of the new structure}

Figure 1 illustrates the transformation process from traditional IDC prototype to two improved structures. From Fig. 1 (a) to (c), the traditional structure is ameliorated step by step. In Fig. 1 (b), previous straight digits in traditional IDC are replaced by curving ones in order to increase the coupling region and consequently promise a larger capacitance. Meanwhile, the number of digits is fixed and the etched area and spaces between two adjacent digits are kept the same. Figure 1 (c) presents the further improved structure comparing with (b). The above three IDC are placed on the same Al Ceramic substrate with

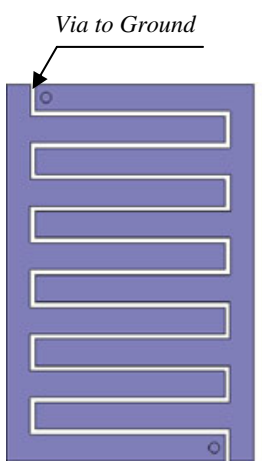

a

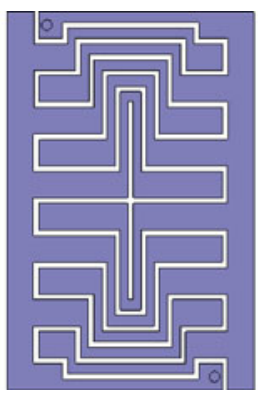

b

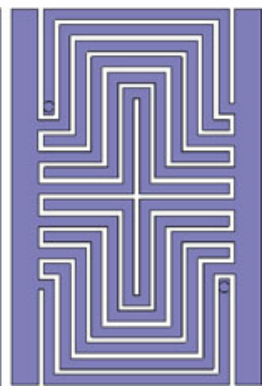

C

Fig. 1 Structure layouts of three CRLH-TL unit cells using different IDC. a Conventional IDC. b, c Two new types of IDC derived from the conventional structure. 
9.8 relative permittivity and $0.5 \mathrm{~mm}$ thickness, having connected to $50 \Omega$ port input and output lines. Physical parameters of the three structures are summarized in Table 1.

\subsection{Equivalent circuit model}

The single unit cell of CRLH-TL is modeled by equivalent LC $\pi$-network as depicted in Fig. 2. The $[\mathrm{ABCD}]$ matrix for TL with a length of $d$ is given by

$$
\left[\begin{array}{ll}
A & B \\
C & D
\end{array}\right]=\left[\begin{array}{ll}
\cosh (\gamma d) & \sinh (\gamma d) / Y_{0} \\
Y_{0} \sinh (\gamma d) & \cosh (\gamma d)
\end{array}\right]
$$

where $Y_{0}$ is the TL's characteristic admittance and $\gamma$ is the propagation constant given by $\gamma=\alpha+j \beta . \alpha$ is the attenuation constant of a signal in the transmission structure and $\beta$ is the wave phase constant. The [ABCD] matrix of the $\pi$-network in Fig. 2 (b) can be given by

$$
\left[\begin{array}{ll}
A & B \\
C & D
\end{array}\right]=\left[\begin{array}{ll}
1+Y_{2} / Y_{3} & 1 / Y_{3} \\
\left(Y_{1} Y_{2}+Y_{2} Y_{3}+Y_{3} Y_{1}\right) / Y_{3} & 1+Y_{2} / Y_{3}
\end{array}\right]
$$

where

$$
Y_{1}=Y_{3}=\frac{1}{j \omega \cdot 2 L_{L}}+j \omega \cdot \frac{1}{2} C_{R}
$$

and

$$
Y_{2}=\left(j \omega L_{R}+\frac{1}{j \omega C_{L}}\right)^{-1}
$$

By using $[\mathrm{ABCD}]$ to S-parameter transformation formulas, we obtain

$$
S_{21}=\frac{2 Y_{0} Y_{3}}{Y_{0}^{2}+Y_{0}\left(Y_{1}+Y_{2}+2 Y_{3}\right)+Y_{1} Y_{2}+Y_{2} Y_{3}+Y_{3} Y_{1}}
$$

Combining (3), (4) and (5), we find the LC parameter expression of $S_{21}$ for the circuit model in Fig. 2 as follows

$$
\begin{aligned}
S_{21}= & j Y_{0}\left(\omega C_{R}-\frac{1}{\omega L_{L}}\right) / \\
& \left(Y_{0}^{2}+\frac{C_{L}}{L_{L}\left(1-\omega^{2} L_{R} C_{L}\right)}+\frac{\omega^{2} C_{R} C_{L}}{1-\omega^{2} L_{R} C_{L}}-\frac{\omega^{2} C_{R}^{2}}{4}-\frac{1}{4 \omega^{2} L_{L}^{2}}+\frac{C_{R}}{2 L_{L}}+j Y_{0}\left(\frac{3 \omega C_{R}}{2}-\frac{3}{2 \omega L_{L}}+\frac{\omega C_{L}}{1-\omega^{2} L_{R} C_{L}}\right)\right)
\end{aligned}
$$

Table 1 Summary of the structure parameters of the IDC.

(a)

(b)

(c)

Area

Spaces Between Digits

Via Diameter

Digit Width

$2.35 \mathrm{~mm} \times 3.55 \mathrm{~mm}$

$0.05 \mathrm{~mm}$

$0.1 \mathrm{~mm}$

$0.25 \mathrm{~mm}$

$0.25 \mathrm{~mm}$ for front and end; $0.1 \mathrm{~mm}$ for bend

$0.1 \mathrm{~mm}$ 

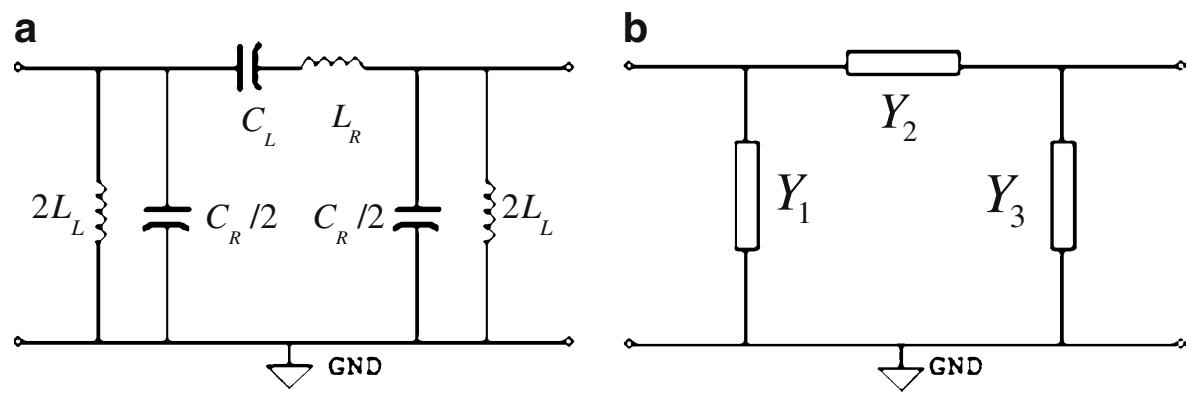

Fig. 2 Equivalent circuit of one unit cell of CRLH-TL. a LC parameter model. $\mathbf{b} \pi$-network model.

\subsection{Full-wave simulation and parameter extraction}

Discussed three types of CRLH-TL are calculated through Ansoft HFSS9 full-wave simulation. Frequency responses described by $S_{11}$ and $S_{21}$ are shown in Fig. 3 (a) and (b). The outlines of the three S-parameter curves are similar, except that they take up different frequency domains, which indicate the levels of intrinsic left-handedness of these three are different. The dispersion relationship for the TL is given by

$$
\cos (\gamma d)=a \sinh (\sqrt{A \cdot D})
$$

Subsequently, the parameter values of $C_{L}, L_{L}, C_{R}$, and $L_{R}$ in (6) are modified by curve fitting of the simulated S-parameter. After applying Bloch-Floquet periodic boundary condition and Kirchhoff's laws [10], the dispersion relationship can be theoretically given

$$
\beta_{B} d=a \cos \left[1-\frac{1}{2}\left(\frac{1}{\omega^{2} L_{L} C_{L}}+\omega^{2} L_{R} C_{R}-\frac{L_{R}}{L_{L}}-\frac{C_{R}}{C_{L}}\right)\right]
$$

Figure 4 illustrates the dispersion relationship calculated from (7) by HFSS and from (8) based on Bloch-Floquet theorem. The LH domain is indicated in the figures, in which the group velocity $v_{g}>0$ and the phase velocity $v_{p}<0$. It is found that the $C_{L}$ values of new type I and II are approximately $38.7 \%$ and $100 \%$ higher than that in the conventional prototype. Also we can discern that the CRLH-TL unit cells employing new types of IDC are capable of operating at greatly lower LH frequency than the traditional structure. Indicated by theoretical calculation, the LH frequency band is spread, however the simulation results show that the bandwidth of Type II is slightly narrower than the other two. The extracted LC parameters and LH frequency bands for the three unit structures are concluded in Table 2.

\section{Implemention of CRLH-TL}

In this section, we design a new 1-D CRLH-TL unit structure using the new type of IDC developed in Section 2 and 3. From the Table 2, it has been found that although the LH frequency region for new type II is pushed down to about $2 \mathrm{GHz}$ lower than the conventional type, its LH bandwidth does not increase as much as new type I. One of the critical elements than limit the bandwidth is the relatively lower value of $L_{L}$ than type I. According to [12], an available method to increase $L_{L}$ so as to spread the bandwidth is 
a

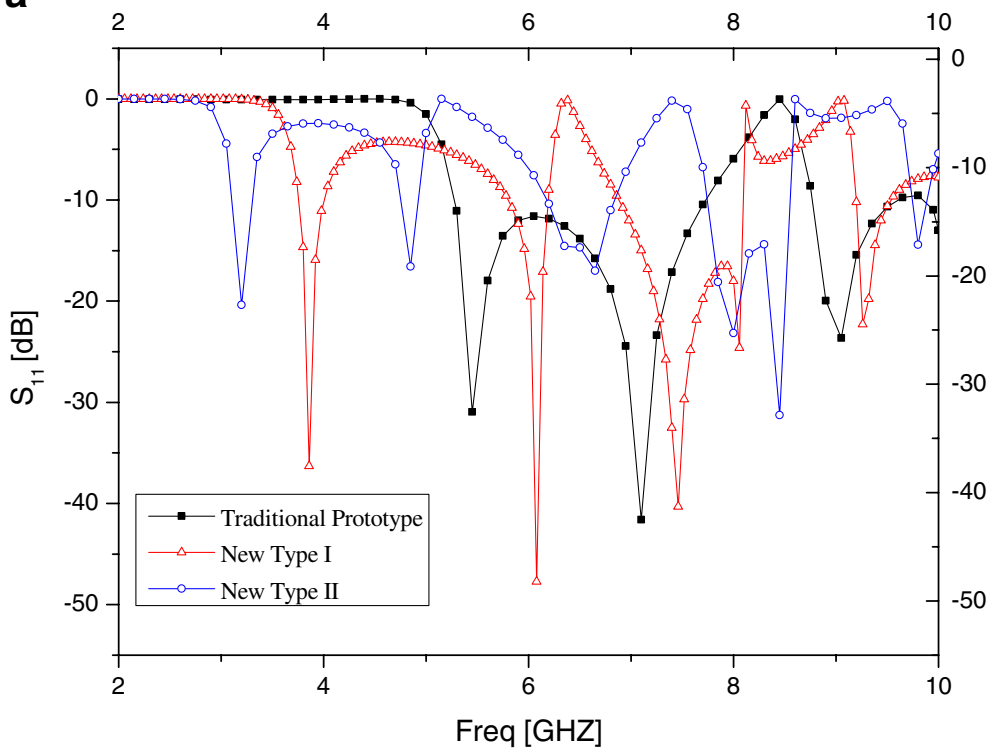

b

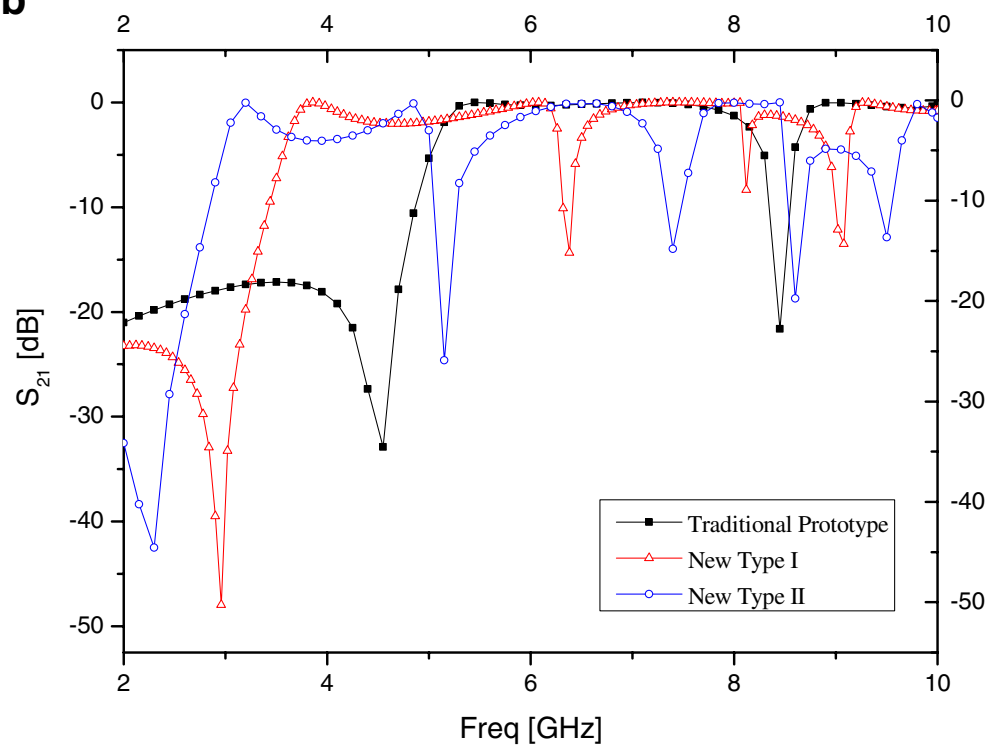

Fig. 3 S-parameter curve calculated by full-wave simulation: (a) $S_{11}$ (b) $S_{21}$.

adopting meandering grounded inductors. The structure of the new CRLH-TL unit structure and its equivalent circuit model are depicted in Fig. 5 (a) and (b). The inderdigital capacitors are the same as the new Type II in above sections. Substrate and all the physical parameters including digits number, widths and spaces between adjacent digits are unaltered. The grounded two vias at two fingertips are removed. Instead, a meandering inductor with a new grounded via at its end is connected to one of the finger. 
a
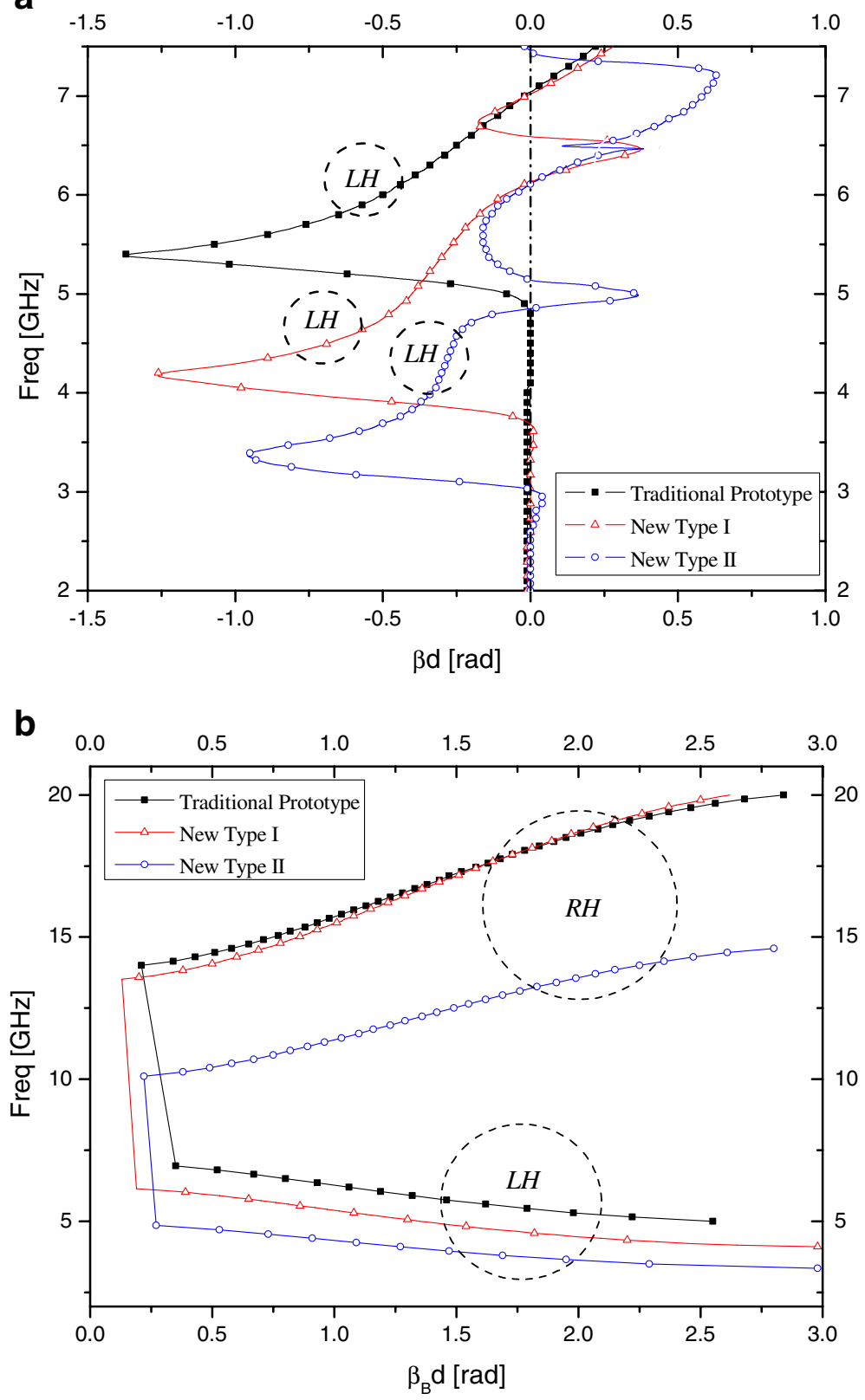

Fig. 4 Dispersion diagram of the three types: (a) Full-wave simulation results (b) Theoretical calculation.

The $[\mathrm{ABCD}]$ matrix of the $T$-network in Fig. 5 (c) can be given by

$$
\left[\begin{array}{ll}
A & B \\
C & D
\end{array}\right]=\left[\begin{array}{ll}
1+Z_{1} / Z_{3} & \left(Z_{1} Z_{2}+Z_{2} Z_{3}+Z_{3} Z_{1}\right) / Z_{3} \\
1 / Z_{3} & 1+Z_{2} / Z_{3}
\end{array}\right]
$$


Table 2 Comparisons of the LC parameters and LH frequency regions of the three types of CRLH-TL.

\begin{tabular}{llll}
\hline & Old Prototype & New Type I & New Type II \\
\hline$C_{L}$ & $0.155 \mathrm{pF}$ & $0.215 \mathrm{pF}$ & $0.31 \mathrm{pF}$ \\
$L_{L}$ & $0.54 \mathrm{nH}$ & $0.92 \mathrm{nH}$ & $0.87 \mathrm{nH}$ \\
$C_{R}$ & $0.168 \mathrm{pF}$ & $0.17 \mathrm{pF}$ & $0.29 \mathrm{pF}$ \\
$L_{R}$ & $3.26 \mathrm{nH}$ & $2.72 \mathrm{nH}$ & $3.39 \mathrm{nH}$ \\
LH Region from Simulation & $5.39 \mathrm{GHz}-7.04 \mathrm{GHz}$ & $4.18 \mathrm{GHz}-6.12 \mathrm{GHz}$ & $3.36 \mathrm{GHz}-4.86 \mathrm{GHz}$ \\
LH Bandwidth from Simulation & $1.65 \mathrm{GHz}$ & $1.94 \mathrm{GHz}$ & $1.78 \mathrm{GHz}$ \\
LH Region from Theory & $5.1 \mathrm{GHz}-6.89 \mathrm{GHz}$ & $4.1 \mathrm{GHz}-6.14 \mathrm{GHz}$ & $3.35 \mathrm{GHz}-4.85 \mathrm{GHz}$ \\
LH Bandwidth from Theory & $1.76 \mathrm{GHz}$ & $2.04 \mathrm{GHz}$ & $1.50 \mathrm{GHz}$ \\
\hline
\end{tabular}

where

$$
Z_{1}=Z_{2}=j \omega \frac{L_{R}}{2}+\frac{1}{j \omega \cdot 2 C_{L}}
$$

and

$$
Z_{3}=\frac{j \omega L_{L}}{1-\omega^{2} L_{L} C_{R}}
$$

Transforming $[\mathrm{ABCD}]$ matrix to $[\mathrm{S}]$ matrix, we obtain

$$
S_{21}=\frac{2 Z_{0} Z_{3}}{Z_{0}+\left(Z_{1}+Z_{2}+2 Z_{3}\right) Z_{0}+Z_{1} Z_{2}+Z_{2} Z_{3}+Z_{3} Z_{1}}
$$
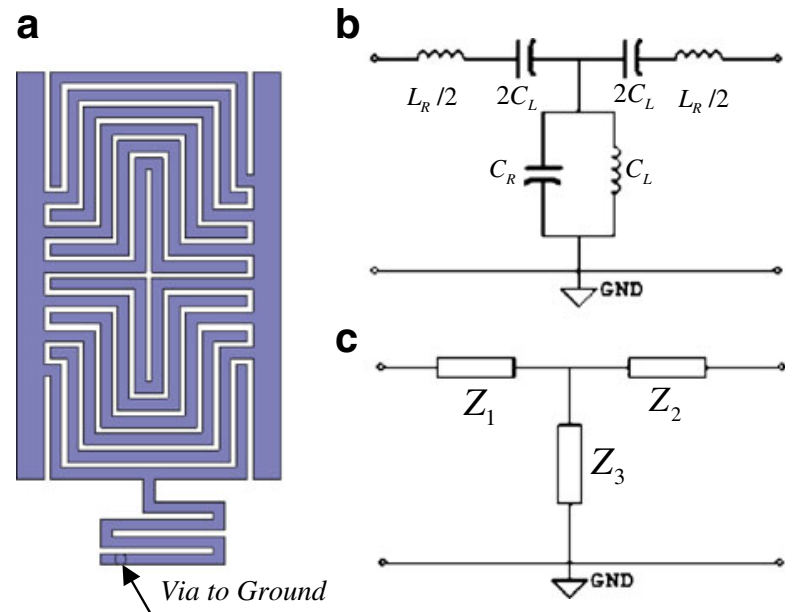

Fig. 5 A new CRLH-TL Unit. a Structure layout. b Equivalent circuit. c T-network. 
a

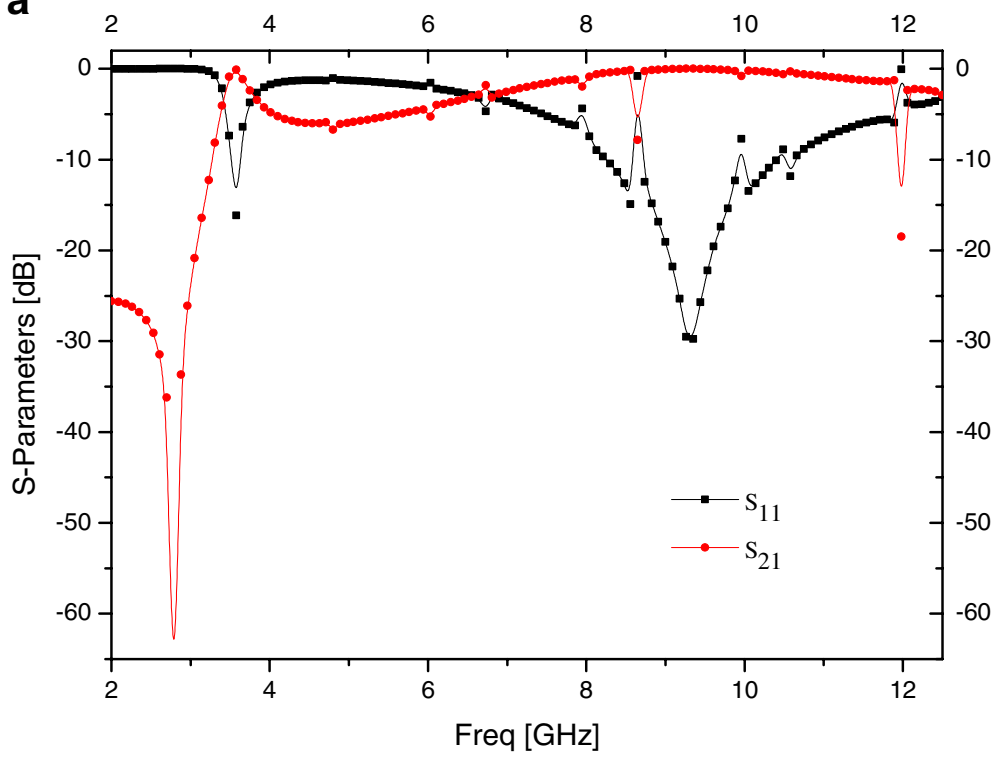

b

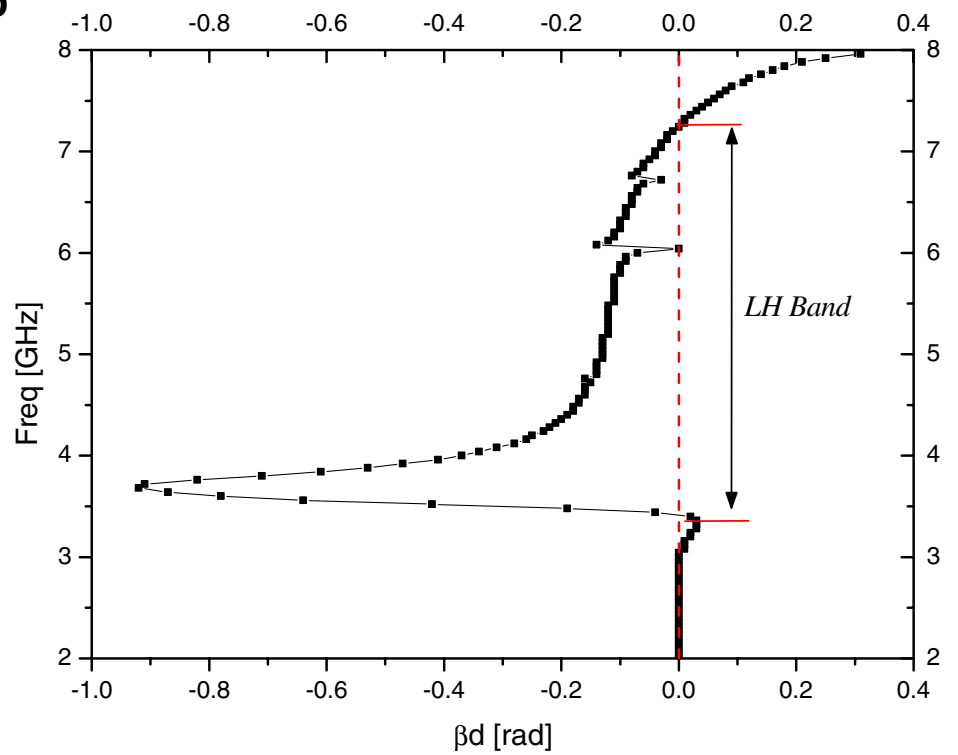

Fig. 6 Simulation results of the CRLH-TL unit structure. a Frequency responses. b Dispersion diagram.

where $Z_{0}$ is the TL's characteristic impedance. With combing (10), (11) and (12), a LC parameter expression of $S_{21}$ is derived as follows

$$
\begin{aligned}
S_{21}= & 2 Z_{0} \cdot j \omega L_{L}\left(1-\omega^{2} L_{L} C_{R}\right)^{-1} / \\
& \left(Z_{0}^{2}+\frac{L_{R}}{2 C_{L}}-\frac{\omega^{2} L_{R}^{2}}{4}+\frac{L_{L}}{C_{L}} \cdot \frac{1}{1-\omega^{2} L_{L} C_{R}}-\frac{\omega^{2} L_{L} L_{R}}{1-\omega^{2} L_{L} L_{R}}-\frac{1}{4 \omega^{2} C_{L}^{2}}+j Z_{0}\left(\omega L_{R}-\frac{1}{\omega C_{L}}+\frac{2 \omega L_{L}}{1-\omega^{2} L_{L} C_{R}}\right)\right)
\end{aligned}
$$


By using the same parameter extraction means in Section 2, the LC values of one unit cell are found: $C_{L}=0.295 \mathrm{pF}, L_{L}=1.09 \mathrm{nH}, C_{R}=0.17 \mathrm{pF}$ and $L_{R}=1.59 \mathrm{nH}$. Frequency response and dispersion relationship are calculated by HFSS9 and illustrated in Fig. 6 (a) and (b). The LH operating frequency band occupies $3.68 \mathrm{GHz}-7.24 \mathrm{GHz}$, with a greatly extended $3.56 \mathrm{GHz}$ bandwidth in contrast with Table 2 in Section 2.

\section{Conclusion}

In this paper, we mainly conduct research on enhancing the LH nature for CRLH-TL unit structure. First, two new types of IDC with much higher capacitance values than conventional one are implemented. Frequency responses are given by full-wave simulation and LC parameters are extracted based on equivalent circuit model. It turns out that the two new types can offer capacitance values approximately $38.7 \%$ and $100 \%$ higher, respectively, than traditional structure. Afterwards dispersion relationships calculated by both HFSS9 and Bloch-Floquet theorem reveal that the proposed new IDC can push the LH frequency band to dramatically lower regions, in the meantime, etched area is kept the same. Then, a novel CRLH-TL unit structure is developed with the improved IDC and meandering grounded inductors. The LH operating band and bandwidth for such new unit cell and traditional one are correspondingly $(3.68 \mathrm{GHz}-7.24 \mathrm{GHz}, 3.56 \mathrm{GHz})$ and (5.39 GHz-7.04 GHz, $1.65 \mathrm{GHz})$, displaying that $1.69 \mathrm{GHz}$ lower and $1.65 \mathrm{GHz}(46 \%)$ wider left-handed frequency band is achieved. This new CRLH-TL unit cell can be periodically cascaded to make up a completed CRLH-TL and also applied in implementation of miniaturized microwave and millimeter-wave devices. In addition, it is feasible to use the proposed types of interdigtal capacitors instead of conventional ones when high capacitance and compact size are demanded.

Open Access This article is distributed under the terms of the Creative Commons Attribution Noncommercial License which permits any noncommercial use, distribution, and reproduction in any medium, provided the original author(s) and source are credited.

\section{References}

1. V. G. Veselago, "The Electrodynamics of Substances with Simultaneously Negative Values of $\varepsilon$ and $\mu$," Soviet Physics Uspekhi, vol. 10, no. 4, pp. 509-514, Jan.-Feb. 1968.

2. R. Smith, W. J. Padilla, D. C. Vier, S. C. Nemat-Nasser, and S. Schultz, "Composite medium with simultaneously negative permeability and permittivity," Phys. Rev. Lett., 84(18): 4184-4187, May 2000 .

3. J. B. Pendry, A. J. Holden, D. J. Robbins, and W. J. Stewart, "Low Frequency Plasmons in thin wire structures," J. Phys. Condense Matter, vol. 10, pp. 4785-809, 1998.

4. J. B. Pendry, A. J. Holden, D. J. Robbins, and W. J. Stewart, "Magnetism from Conductors and Enhanced Nonlinear Phenomena," IEEE Trans. Microwave Theory and Tech, vol. 47, no. 11, pp. 20752084, Nov. 1999.

5. C. G. Parazzoli, R. B. Greegor, K. Li, B. E. C. Koltenbah, and M. Tanielian, "Experimental Verification and Simulation of Negative Index of Refraction Using Snell's Law," Phys. Rev. Lett, 90(10): 0031-9007, Mar. 2003.

6. C. Caloz and T. Itoh, "Transmission line approach of left-handed (LH) materials and microstrip implementation of an artificial LH transmission line, "IEEE Trans. Antenas and Propaga., vol. 52, pp. 2701-2712, Dec. 2002. 
7. G. V. Eleftheriades, A. K. Iyer, and P. C. Kremer, "Planar negative refractive index media using periodically LC loaded transmission lines, "IEEE Trans. Microwave Theory and Tech, Dig., vol. 1, pp. 191-194, Philadelphia, PA, Jun. 2003.

8. C. Cheng and R. W. Ziolkowshi, "Tailoring double-negative metamaterial responses to achieve anomalous propagation effects along microstrip transmission lines," IEEE Trans. Microwave Theory and Tech, vol. 51, no. 12, pp. 2306-2314, Dec. 2003.

9. IEEE Microwave Magazine, pp. 1527-3342, Sept. 2004.

10. C Caloz, A. Sanada, and T. Itoh, "A Novel Composite Right-/Left-Handed Coupled-Line Directional Coupler with arbitrary Couping leve and Broad Bandwidth," IEEE Trans. Microwave Theory and Tech, vol. 52, no. 3, pp. 0018-9480, Mar. 2004.

11. Shau-Gang Mao, and Yu-Zhi Chueh, "Broadband Composite Right/Left-Handed" Coplanar Waveguide Power Splitter With Arbitrary Phase Responses and Balun and Antenna Applications", IEEE Trans. Antenas and Propaga., vol. 54, no. 1, pp. 0018-926X, Jan. 2006.

12. Atsushi Sanada, Koichi Murakami, Shuji Aso, Hiroshi Kubo, and kuo Awai, "A Via-Free Microstrip Left-Handed Transmission Line", IEEE International Microwave Symposium Proceedings, Fort Worth, Jun. 2004 